\title{
Solution of Earth orientation parameters in 20th century based on optical astrometry and new catalog EOC-3
}

\author{
J. Vondrák, C. Ron and V. Štefka \\ Astronomical Institute, \\ Boční II, CZ-14131, Prague, Czech Republic \\ email: vondrak@ig.cas.cz
}

\begin{abstract}
A new star catalog has been derived recently, which is based on combination of Hipparcos/Tycho Catalogues with long lasting ground-based astrometric observations. In addition to 'classical' mean positions and proper motions of the stars, it contains also information on periodic motions of many stars that are due to double or multiple stellar systems. The catalog, called EOC-3, contains 4418 different objects, out of which 585 have significant periodic motions. This improved catalog is used as a reference frame in optical wavelength to derive a new series of Earth Orientation Parameters. To this end, almost five million observations of latitude/universal time variations made at 33 observatories are used. The new EOP series covers almost the entire 20th century (namely the interval 1899.7-1992.0).
\end{abstract}

Keywords. astrometry, catalogs, reference systems

\section{Introduction}

Some years ago, we collected the astrometric observations of latitude/universal time variations made worldwide at 33 observatories. These observations, re-analyzed with the Hipparcos Catalogue, were then used to determine the Earth Orientation Parameters (EOP) at 5-day intervals, covering the interval 1899.7-1992.0 (Vondrák et al. 1998, Vondrák et al. 2000, Ron et al. 2005). The observations, accumulated during almost a century of monitoring the Earth orientation, contain a valuable and rich astrometric material that was only seldom used to construct astrometric catalogs. Later on, new astrometric catalogues such as ARIHIP (Wielen et al. 2001) or TYCHO-2 (Høg et al. 2000) appeared as combination of Hipparcos/Tycho positions with ground-based catalogues. These catalogues yield more accurate proper motions than the original Hipparcos Catalogue.

Many of the objects observed in the programmes of monitoring Earth orientation from the ground are double or multiple systems, having non-negligible periodic motions. We tried to obtain a star catalogue with improved proper motions and quasi-periodic terms reflecting orbital motions of the stars observed in these programmes. To this end, we used about 4.5 million observations of latitude/universal time variations, and combined them with the catalogues ARIHIP, TYCHO-2, to obtain a new astrometric catalog, 'tailored' for long-term Earth orientation studies. The third version of the Earth Orientation Catalogue (EOC-3) contains 4418 different objects (i.e., stars, components of double stars, photocenters), out of which 585 have statistically significant periodic motions (Vondrák \& Štefka 2007). Namely this catalog is used in the solution whose description follows. 


\section{The solution}

Observations of variations of latitude $(\Delta \varphi)$, universal time (UT0-UTC) and equal altitude differences $\delta h$ with 47 different instruments, located at 33 observatories, were first recalculated with the new astrometric catalogue EOC-3, nutation IAU2000 (Mathews et al. 2002) and precession IAU2006 (Capitaine et al. 2003), and then used to determine Earth Orientation Parameters (EOP) at 5 -day intervals: Coordinates of the pole in terrestrial frame $x, y$, and Universal time differences UT1-TAI (only after 1956).

For each instrument, we determine constant, linear, annual and semi-annual deviation in latitude / longitude $\operatorname{dev}_{\varphi}, \operatorname{dev}_{\lambda}$, and rheological parameter (combination of Love numbers) $\Lambda=1+k-l$ for the tidal variations of the vertical.

For the whole interval, we determine celestial pole offsets with respect to IAU2000/2006 precession-nutation model $\mathrm{d} X, \mathrm{~d} Y$, represented as quadratic functions of time.

Data from the following instruments were used:

- 10 PZT's (measuring $\Delta \varphi$, UT0-UTC): 3 at Washington; 2 at Richmond and Mizusawa; 1 at Mount Stromlo, Punta Indio \& Ondřejov;

- 7 photoelectric transit instruments (measuring only UT0-UTC): 3 at Pulkovo; 1 at Irkutsk, Kharkov, Nikolaev \& Wuhang;

- 16 visual zenith-telescopes \& similar instruments (measuring $\Delta \varphi$ only): 7 ZT at ILS stations; 2 ZT at Poltava, 1 ZT at Belgrade, Blagoveschtchensk, Irkutsk, Jósefoslaw \& Pulkovo; FZT at Mizusawa; VZT at Tuorla-Turku;

- 14 instruments for equal altitude observations - AST, PAST, CZ (measuring $\delta h$ ): 1 AST at Paris, Santiago de Chile, Shanghai, Simeiz \& Wuhang; 2 PAST at Shaanxi, 1 PAST at Beijing, Grasse, Shanghai \& Yunnan; 1 CZ at Bratislava, Prague \& Pecný.

The geographic distribution of all 33 participating observatories is depicted in Figure 1.

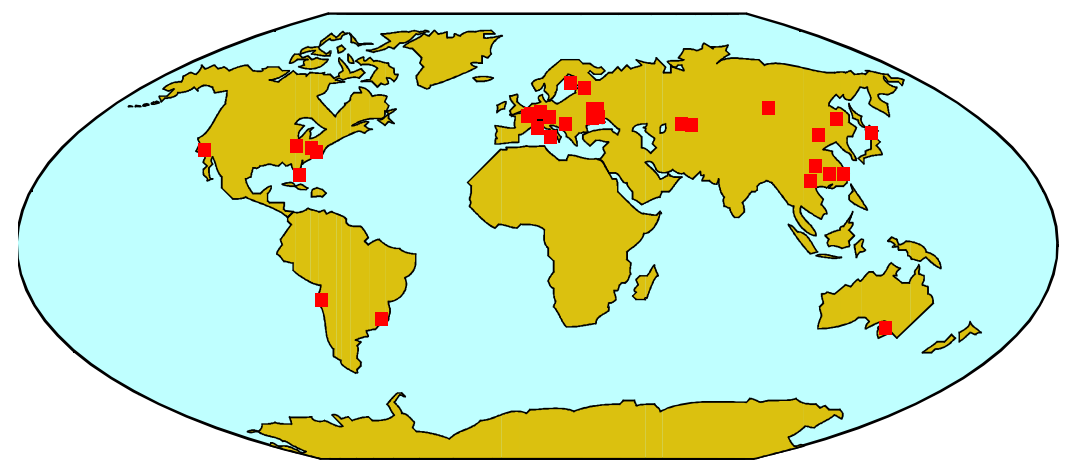

Figure 1. Geographic distribution of participating observatories.

To derive the EOP's, we use a procedure very similar to the one used earlier (Vondrák et al. 1998), i.e., we used the observation equations, which in a simplified form read

$$
\Delta \varphi=x \cos \lambda-y \sin \lambda-d X \cos \alpha-d Y \sin \alpha+d e v_{\varphi}+\Lambda D_{\varphi},
$$

$15 \cos \varphi(\mathrm{UT} 0-\mathrm{UTC})=15 \cos \varphi(\mathrm{UT} 1-\mathrm{UTC})+\sin \varphi(x \sin \lambda+y \cos \lambda)+$

$$
\begin{aligned}
& +\cos \varphi \tan \delta(d Y \cos \alpha-d X \sin \alpha)+d e v_{\lambda}+15 \Lambda D_{\lambda} \cos \varphi, \\
\delta h & =15 \cos \varphi \sin a(\mathrm{UT} 1-\mathrm{UTC})+x(\cos \lambda \cos a+\sin \varphi \sin \lambda \sin a)- \\
& -y(\sin \lambda \cos a-\sin \varphi \cos \lambda \sin a)+d Y(\sin q \sin \delta \cos \alpha-\cos q \sin \alpha)- \\
& -d X(\sin q \sin \delta \sin \alpha+\cos q \cos \alpha)+d e v_{\varphi} \cos a+d e v_{\lambda} \sin a+ \\
& +\Lambda\left(D_{\varphi} \cos a+15 D_{\lambda} \cos \varphi \sin a\right),
\end{aligned}
$$


where $\varphi, \lambda$ are the observatory's geographic coordinates, $\alpha, \delta, a$ and $q$ are right ascension, declination, azimuth and parallactic angle of the star, respectively, and $D_{\varphi}, D_{\lambda}$ are tidal variations of the local vertical computed for rigid Earth.
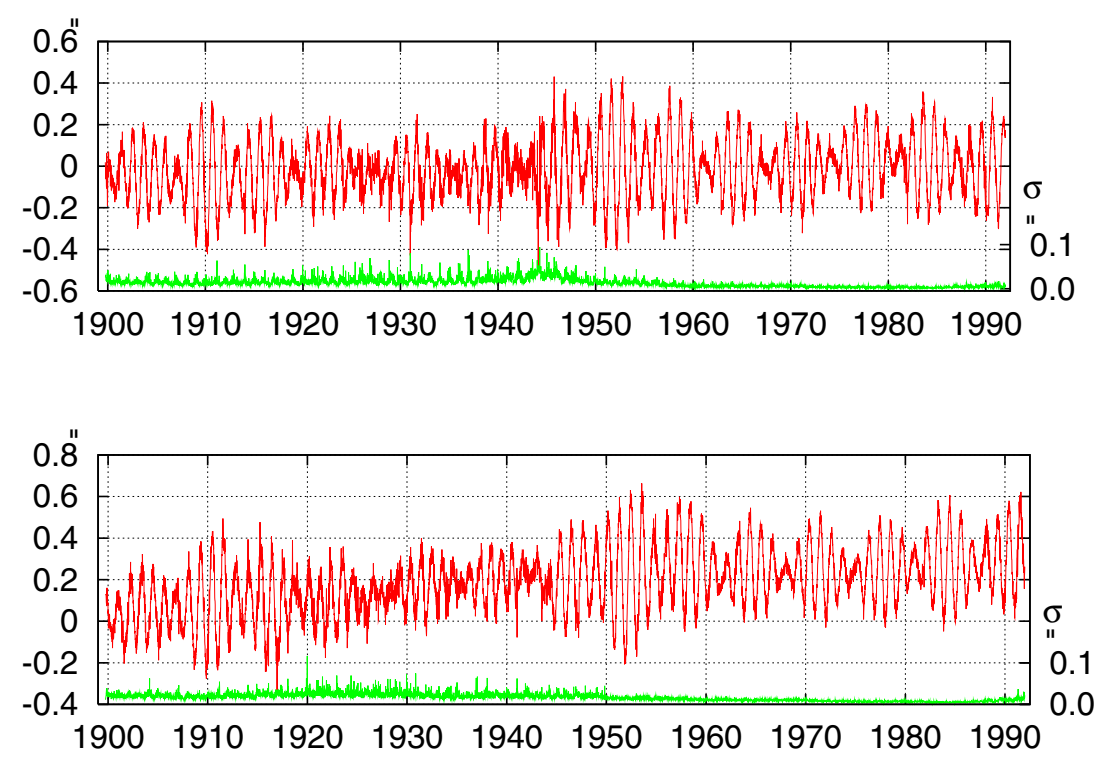

Figure 2. Polar motion $x$ (upper plot), $y$ (lower plot), and its uncertainties.

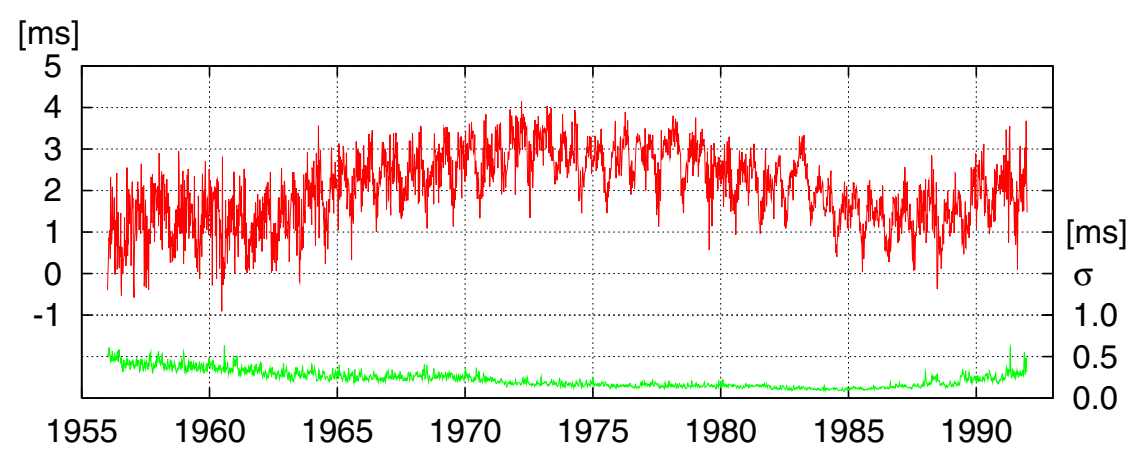

Figure 3. Length-of-day without short-period tidal variations, and its uncertainties.

The results are displayed in Figures 2-3, where both components of polar motion $x, y$ and length-of day changes, calculated as the rate of UT1-UTC, are given. In the latter, the tidal variations after Yoder et al. (1981), with periods shorter than 35 days, were removed. The rheological parameters $\Lambda$, calculated for all participating observatories, are shown in Figure 4. The observatories are ranged by their increasing longitudes.

The celestial pole offsets, expressed with respect to IAU2000 model of nutation and IAU2006 model of precession, are very small so that it is impossible to detect their quasi-periodic variations from optical astrometry. Therefore they were estimated only as quadratic functions of time (in milliarcseconds):

$$
\begin{aligned}
& d X=-7.4+28.6 T+30.0 T^{2} \\
& d Y=-5.9+9.4 T+1.2 T^{2}
\end{aligned}
$$




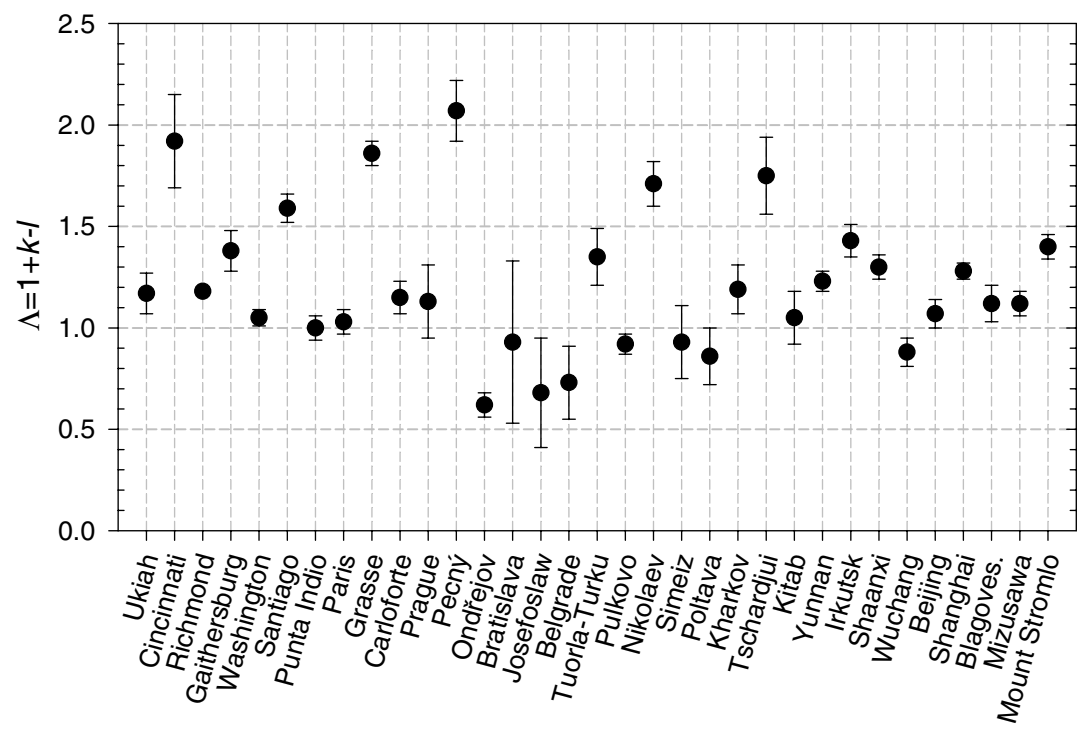

Figure 4. Rheological parameter $\Lambda=1+k-l$.

where $T$ counts in centuries from 1956.0. The uncertainties of constant, linear and quadratic terms are $\pm 0.4 \mathrm{mas}, 1.1 \mathrm{mas} / \mathrm{cy}$ and $3.5 \mathrm{mas} / \mathrm{cy}^{2}$, respectively.

\section{Conclusions}

The new solution, based on catalog EOC-3, used 4541706 individual observations. It yields slightly better results than the one based on its previous version, EOC-2 (Vondrák 2004 ); the average standard error of one observation is $\sigma_{\circ}=0.190^{\prime \prime}$ (in contrast to its previous value $\left.0.191^{\prime \prime}\right)$. The results are quite similar, especially the celestial pole offsets, given by Eqs. (2.2), still contain rather large linear and quadratic terms compatible with those found in (Ron et al. 2005). The new solution will serve for further long-term Earth rotation studies.

\section{References}

Capitaine, N., Wallace, P. T., \& Chapront, J. 2003, A\&A, 412, 567

$\mathrm{H} ø$ g, E., Fabricius, C., Makarov, V. V. et al. 2000, A\& A, 355, L27

Mathews, P. M., Herring, T. A., \& Buffet, B. A. 2002, J. Geophys. Res., 107 (B4), doi: 10.1029/2001JB000390.

Ron, C., Capitaine, N., \& Vondrák, J. 2005, in: N. Capitaine (ed.) Journées 2004 Systèmes de référence spatio-temporels, (Observatoire de Paris), p. 110

Vondrák, J. 2004, Serb. Astron. J. 168, 1

Vondrák, J. \& Štefka, V. 2007, A\&SA, 463, 783

Vondrák, J., Pešek, I., Ron, C., \& Čepek, A. 1998, Publ. Astron. Inst. Acad. Sci. Czech R. 87

Vondrák, J., Ron, C., \& Pešek, I. 2000, in: S. Dick, D. D. McCarthy, \& B. Luzum (eds.), Polar motion: Historical and Scientific Problems, Proc. IAU Coll. 178, ASP Conf. Series 208, p. 206

Wielen, R., Schwan, H., Dettbarn, C. et al. 2001, Veröff. Astron. Rechen-Inst. Heidelberg, 40, Kommissions-Verlag G. Braun, Karlsruhe.

Yoder, C. F., Williams, J. G., \& Parke, M. E. 1981, J. Geophys. Res. 86, 881 